\title{
RECTANGULAR DIFFERENTIATION OF INTEGRALS OF BESOV FUNCTIONS
}

\author{
Hugo Aimar, Liliana Forzani, and Virginia Naibo
}

\begin{abstract}
We study the differentiation of integrals of functions in the Besov spaces $B_{p}^{\alpha, 1}\left(\mathbb{R}^{n}\right), \alpha>0,1 \leq p<\infty$, with respect to the basis of arbitrarily oriented rectangular parallelepipeds in $\mathbb{R}^{n}$. We show that positive results hold if $\alpha \geq \frac{n-1}{p}$ and we give counterexamples for the case $0<\alpha<\frac{n}{p}-1$. Similar results hold for $B_{p}^{\alpha, q}\left(\mathbb{R}^{n}\right), q>1$. For more general bases we can also prove negative results for $\frac{n}{p}-1 \leq \alpha<\frac{n-1}{p}$.
\end{abstract}

\section{Definitions and main results}

Following de Guzmán [3,4] we shall say that a differentiation basis $\mathcal{A}$ is a collection of open bounded sets in $\mathbb{R}^{n}$ such that for each $x \in \mathbb{R}^{n}$ there is a sequence $\left\{A_{j}\right\} \subset \mathcal{A}$ with $x \in A_{j}$ for every $j$ and diameter of $A_{j}$ tending to 0 .

A differentiation basis $\mathcal{A}$ is said to differentiate the integral of a locally integrable function $f$ defined in $\mathbb{R}^{n}$ if

$$
\lim _{\operatorname{diam}(A) \rightarrow 0, x \in A \in \mathcal{A}} \frac{1}{|A|} \int_{A} f(y) d y=f(x)
$$

for almost every $x \in \mathbb{R}^{n}$, where $|A|$ denotes the Lebesgue measure of the set $A$. If $\mathcal{A}$ differentiates the integral of every function of a given class we say that $\mathcal{A}$ differentiates that class.

We denote by $\mathcal{B}$ the basis of all arbitrarily oriented rectangular parallelepipeds in $\mathbb{R}^{n}$ with diameter smaller than 1 . Each element of $\mathcal{B}$ can be regarded as a proper rigid motion of a multidimensional interval of the form $\prod_{j=1}^{n}\left(-b_{j}, b_{j}\right)$, $b_{j}>0$ and $\sum_{j=1}^{n}\left(2 b_{j}\right)^{2} \leq 1$.

The basis $\mathcal{B}$ does not differentiate the spaces $L^{p}\left(\mathbb{R}^{n}\right)$. Actually, it does not even differentiate the characteristic functions of measurable sets in $\mathbb{R}^{n}$, as was observed by Zygmund (in Nikodym [6]) (see [3, 8]). Then, no restriction on the global growth of functions is sufficient for differentiation of integrals with respect to that basis and it seems natural to impose additional restrictions on the integral smoothness of functions. In that direction, Stokolos $[9,10]$ considered the

Received November 2, 2001.

2000 Mathematics Subject Classification. 42B35, 42B25.

Key words and phrases. Differentiation, Besov spaces, maximal functions.

Supported by CONICET and Universidad Nacional del Litoral. 
differentiation of the integral of functions in terms of the integrability properties of the $L^{p}$ modulus of continuity.

We define the Besov spaces $B_{p}^{\alpha, q}\left(\mathbb{R}^{n}\right)$ for $\alpha>0$ and $1 \leq p, q \leq \infty$. Let $\bar{\alpha}$ be the smallest integer greater than $\alpha$ and denote by $u_{f}(x, t)=\left(f * P_{t}\right)(x), x \in \mathbb{R}^{n}$, $t>0$, the Poisson integral of $f \in L^{p}\left(\mathbb{R}^{n}\right)$, where $P_{t}(x)=c_{n} t /\left(t^{2}+|x|^{2}\right)^{(n+1) / 2}$, $c_{n}=\Gamma((n+1) / 2) / \pi^{(n+1) / 2}$. Then $B_{p}^{\alpha, q}\left(\mathbb{R}^{n}\right)$ is the set of functions for which the norm

$$
\|f\|_{B_{p}^{\alpha, q}\left(\mathbb{R}^{n}\right)}=\|f\|_{L^{p}\left(\mathbb{R}^{n}\right)}+\left(\int_{0}^{\infty}\left\|t^{\bar{\alpha}-\alpha} \frac{\partial^{\bar{\alpha}} u_{f}}{\partial t^{\bar{\alpha}}}(\cdot, t)\right\|_{L^{p}\left(\mathbb{R}^{n}\right)}^{q} \frac{d t}{t}\right)^{\frac{1}{q}}
$$

is finite (with the obvious changes for $q=\infty$ ).

In this paper we study the differentiation of integrals of functions in $B_{p}^{\alpha, q}\left(\mathbb{R}^{n}\right)$ with respect to $\mathcal{B}$. The problem only has interest when $n \geq 2$ and $p<\infty$ since otherwise we are dealing with intervals or continuous functions. The main results are the following:

Theorem 1.2.

a) If $1 \leq p<\infty$ then $\mathcal{B}$ differentiates $B_{p}^{(n-1) / p, 1}\left(\mathbb{R}^{n}\right)$.

b) If $1 \leq p<n$ and $0<\alpha<\frac{n}{p}-1$ then $\mathcal{B}$ does not differentiate $B_{p}^{\alpha, 1}\left(\mathbb{R}^{n}\right)$.

Using the immersion theorems for Besov spaces we can extend the positive and negative results in the following way:

\section{Corollary 1.3.}

a) Let $1 \leq p<\infty$. If either $1<q \leq \infty$ and $\alpha>\frac{n-1}{p}$ or $q=1$ and $\alpha \geq \frac{n-1}{p}$ then $\mathcal{B}$ differentiates $B_{p}^{\alpha, q}\left(\mathbb{R}^{n}\right)$.

b) If $1 \leq p<n, 0<\alpha<\frac{n}{p}-1$ and $1 \leq q \leq \infty$ then $\mathcal{B}$ does not differentiate $B_{p}^{\alpha, q}\left(\mathbb{R}^{n}\right)$.

Let us point out that we do not have a result for $\frac{n}{p}-1 \leq \alpha<\frac{n-1}{p}$.

Part $a$ ) of Theorem 1.2 will follow from the next local weak type inequality:

Theorem 1.4. Let $\mathcal{M}$ be the maximal operator associated to $\mathcal{B}$,

$$
\mathcal{M} f(x)=\sup _{x \in R \in \mathcal{B}} \frac{1}{|R|} \int_{R}|f(y)| d y .
$$

If $1 \leq p<\infty$ and $r>0$ then

$$
\left|\left\{x \in \mathbb{R}^{n}: \mathcal{M} f(x)>\lambda\right\}\right| \leq \frac{c}{\lambda}(r+1)^{n-1} r^{1 / p^{\prime}}\|f\|_{B_{p}^{(n-1) / p, 1}\left(\mathbb{R}^{n}\right)}
$$

for every $f \in B_{p}^{(n-1) / p, 1}\left(\mathbb{R}^{n}\right)$ with $\sup p(f) \subset B(0, r)=\left\{x \in \mathbb{R}^{n}:|x|<r\right\}$. Here $c$ is a constant independent of $f$ and $r$ and $\frac{1}{p}+\frac{1}{p^{\prime}}=1$.

Part $b$ ) of Corollary 1.3 can be improved to get the following result: 
Theorem 1.6. If $1 \leq p<n, 0<\alpha<\frac{n}{p}-1$ and $1 \leq q \leq \infty$, then there exists $f \in B_{p}^{\alpha, q}\left(\mathbb{R}^{n}\right)$ such that

$$
\limsup _{\operatorname{diam}(R) \rightarrow 0, x \in R \in \mathcal{B}} \frac{1}{|R|} \int_{R} f(y) d y=+\infty
$$

for every $x \in Q_{0}=\left\{y=\left(y_{1}, \cdots, y_{n}\right):-\frac{1}{2}<y_{i} \leq \frac{1}{2}, i=1, \cdots, n\right\}$.

In $\S 2$ we enumerate different equivalent norms in $B_{p}^{\alpha, q}\left(\mathbb{R}^{n}\right)$ that we will use throughout this paper and we state immersion and regularization results for Besov spaces. In $\S 3$ we prove a trace inequality which is interesting by itself and will be useful in the proof of Theorem 1.4. In $\S 4$ we give the proofs of parts a) of Theorem 1.2 and Corollary 1.3 (positive results). In $\S 5$ we present the proofs of parts $b$ ) of Theorem 1.2 and Corollary 1.3 and Theorem 1.6 (negative results). In $\S 6$ we consider the differentiation of integrals of Besov functions with respect to a somehow more general basis. In particular we show that the gap $\frac{n}{p}-1 \leq \alpha<\frac{n-1}{p}$ is filled with negative results.

In what follows, $c$ will denote a constant that can vary even within a single chain of inequalities.

\section{Norms, inmersions, regularization and localization of besov functions}

Throughout this paper we will use different norms equivalent to (1.1) in $B_{p}^{\alpha, q}\left(\mathbb{R}^{n}\right)$ :

1. For $k$ an integer larger than $\alpha$, an equivalent norm is given by

$$
\|f\|_{L^{p}\left(\mathbb{R}^{n}\right)}+\left(\int_{0}^{\infty}\left\|t^{k-\alpha} \frac{\partial^{k} u_{f}}{\partial t^{k}}(\cdot, t)\right\|_{L^{p}\left(\mathbb{R}^{n}\right)}^{q} \frac{d t}{t}\right)^{\frac{1}{q}} .
$$

(Taibleson [11]).

2. For $k \in \mathbb{N}$ and $f \in L^{p}\left(\mathbb{R}^{n}\right)$ we introduce the $L^{p}$ modulus of continuity of $f$ of order $k$,

$$
\omega_{k}(f, t)_{p}=\sup _{|h| \leq t, h \in \mathbb{R}^{n}}\left\|\Delta_{h}^{k} f\right\|_{L^{p}\left(\mathbb{R}^{n}\right)}
$$

where $\Delta_{h}^{k} f(x)=\sum_{i=0}^{k}\left(\begin{array}{c}k \\ i\end{array}\right)(-1)^{k-i} f(x+i h)$ is the finite difference operator of order $k$ and step $h$. Then, if $k>\alpha$,

$$
\|f\|_{L^{p}\left(\mathbb{R}^{n}\right)}+\left(\int_{0}^{\infty}\left(t^{-\alpha} \omega_{k}(f, t)_{p}\right)^{q} \frac{d t}{t}\right)^{\frac{1}{q}}
$$

is an equivalent norm in $B_{p}^{\alpha, q}\left(\mathbb{R}^{n}\right)$ (Triebel [12]). 
3. Let $\varphi \in \mathcal{S}\left(\mathbb{R}^{n}\right)$, the Schwartz class, be such that $\operatorname{supp}(\hat{\varphi}) \subset\{1 / 2 \leq|\xi| \leq 2\}$ and $|\hat{\varphi}(\xi)| \geq c$ for $3 / 5 \leq|\xi| \leq 5 / 3$ for some constant $c>0$, where $\hat{\varphi}$ denotes the Fourier transform of $\varphi$. Another equivalent norm in $B_{p}^{\alpha, q}\left(\mathbb{R}^{n}\right)$ is given by

$$
\|f\|_{L^{p}\left(\mathbb{R}^{n}\right)}+\left(\int_{0}^{\infty}\left(t^{-\alpha}\left\|\varphi_{t} * f\right\|_{L^{p}\left(\mathbb{R}^{n}\right)}\right)^{q} \frac{d t}{t}\right)^{1 / q}
$$

where $\varphi_{t}(x)=t^{-n} \varphi(x / t)$ (Triebel [12],Bui-Paluszyński-Taibleson [2]).

In (2.7), (2.8) and (2.9) we have the obvious changes for $q=\infty$ and if $q<\infty$, $\int_{0}^{\infty}$ may be replaced by $\int_{0}^{1}$.

We have the following immersion results for Besov spaces (Taibleson [11]):

Proposition 2.10. $B_{p}^{\alpha, q}\left(\mathbb{R}^{n}\right) \hookrightarrow B_{w}^{\beta, v}\left(\mathbb{R}^{n}\right)$ if and only if either $p \leq w$ and $\alpha-\frac{n}{p}>\beta-\frac{n}{w}$ or $p \leq w, \alpha-\frac{n}{p}=\beta-\frac{n}{w}$ and $q \leq v$.

Localization and regularization are two valid procedures in Besov spaces. The following lemma will be helpful in the proofs of the results in $\S 4$ (see Taibleson [11] and Triebel [12]). In the following if $\nu=\left(\nu_{1}, \cdots, \nu_{n}\right) \in \mathbb{N}_{0}^{n}$ is a multiindex then $|\nu|=\sum_{i=1}^{n} \nu_{i}$ and $D^{\nu} g$ denotes the derivative of order $\nu$ of $g . \mathcal{C}_{0}^{\infty}\left(\mathbb{R}^{n}\right)$ is the set of indefinitely differentiable functions on $\mathbb{R}^{n}$, with compact support.

Lemma 2.11. Let $\alpha>0$ and $1 \leq p, q \leq \infty$.

a) If $p, q<\infty$ and $f \in B_{p}^{\alpha, q}\left(\mathbb{R}^{n}\right)$ with $\operatorname{supp}(f) \subset B(0, r), r>0$, then, given $\epsilon>0$, there exists $g \in \mathcal{C}_{0}^{\infty}\left(\mathbb{R}^{n}\right)$ such that $\operatorname{supp}(g) \subset B(0,2 r)$ and $\| f-$ $g \|_{B_{p}^{\alpha, q}\left(\mathbb{R}^{n}\right)}<\epsilon$.

b) If $g \in \mathcal{C}_{0}^{\infty}\left(\mathbb{R}^{n}\right)$ then $g f \in B_{p}^{\alpha, q}\left(\mathbb{R}^{n}\right)$ for every $f \in B_{p}^{\alpha, q}\left(\mathbb{R}^{n}\right)$. Moreover if $m \in \mathbb{N}, m>\alpha$, then

$$
\|g f\|_{B_{p}^{\alpha, q}\left(\mathbb{R}^{n}\right)} \leq c \sum_{|\nu| \leq m}\left\|D^{\nu} g\right\|_{L^{\infty}\left(\mathbb{R}^{n}\right)}\|f\|_{B_{p}^{\alpha, q}\left(\mathbb{R}^{n}\right)} .
$$

where $c$ is a constant independent of $f$ and $g$.

c) We have $\mathcal{C}_{0}^{\infty}\left(\mathbb{R}^{n}\right) \subset B_{p}^{\alpha, q}\left(\mathbb{R}^{n}\right)$. Moreover, if $p, q<\infty$ then $\mathcal{C}_{0}^{\infty}\left(\mathbb{R}^{n}\right)$ is a dense subset in $B_{p}^{\alpha, q}\left(\mathbb{R}^{n}\right)$.

\section{A trace inequality}

If $x \in \mathbb{R}^{n}$ we set $x=\left(x^{\prime}, x_{n}\right)$ where $x^{\prime} \in \mathbb{R}^{n-1}$ and $x_{n} \in \mathbb{R}$. For a function $f$ of variable $x \in \mathbb{R}^{n}$, we write $\left\|f\left(\cdot, x_{n}\right)\right\|_{B_{p}^{\alpha, 1}\left(\mathbb{R}^{n-1}\right)}$ for the norm in $B_{p}^{\alpha, 1}\left(\mathbb{R}^{n-1}\right)$ of $f\left(x^{\prime}, x_{n}\right)$ as a function of $x^{\prime}$.

In this section we are going to prove the following theorem:

Theorem 3.12. Let $\alpha>0,1 \leq p \leq \infty, r>0$ and $f \in \mathcal{C}_{0}^{\infty}\left(\mathbb{R}^{n}\right)$. If $\operatorname{supp}(f) \subset$ $B(0, r)$ then

$$
\int_{-r}^{r}\left\|f\left(\cdot, x_{n}\right)\right\|_{B_{p}^{\alpha, 1}\left(\mathbb{R}^{n-1}\right)} d x_{n} \leq c r^{1 / p^{\prime}}\|f\|_{B_{p}^{\alpha, 1}\left(\mathbb{R}^{n}\right)}
$$

where $c$ is a constant independent of $f$ and $r$. 
For the proof of Theorem 3.12 we need the following lemmata:

Lemma 3.13. If $f$ is an indefinitely differentiable function on $\mathbb{R}^{n}, k \in \mathbb{N}$ and $h \in \mathbb{R}^{n}$ then

$$
\Delta_{h}^{k} f(x)=\int_{\mathbb{R}} \sum_{|\nu|=k} \frac{k !}{\nu !}\left(D^{\nu} f\right)(x+\xi h) h^{\nu} M_{k}(\xi) d \xi,
$$

where $M_{1}=\chi_{(0,1)}$ and $M_{k}=M_{1} * M_{k-1}$ for $k \geq 2$. Here $h^{\nu}=h_{1}^{\nu_{1}} \cdots h_{n}^{\nu_{n}}$ and $\nu !=\nu_{1} ! \cdots \nu_{n} !$ if $h=\left(h_{1}, \cdots, h_{n}\right)$ and $\nu=\left(\nu_{1}, \cdots, \nu_{n}\right)$.

Lemma 3.14. If $1 \leq p \leq \infty$ and $f \in \mathcal{C}_{0}^{\infty}\left(\mathbb{R}^{n}\right)$ then $u_{f}\left(x^{\prime}, x_{n}, y\right) \rightarrow f\left(x^{\prime}, x_{n}\right)$ in $L^{p}\left(\mathbb{R}^{n-1}\right)$ as $y \rightarrow 0$ for every $x_{n} \in \mathbb{R}$.

Proof of Theorem 3.12. Fix $r>0, \alpha>0,1 \leq p \leq \infty$ and $k \in \mathbb{N}, k>\alpha$.

Let $f \in \mathcal{C}_{0}^{\infty}\left(\mathbb{R}^{n}\right)$ with $\operatorname{supp}(f) \subset B(0, r), y, t>0, y<t$, and consider the following integral version of Taylor's formula

$$
\begin{aligned}
u_{f}\left(x^{\prime}, x_{n}, y\right) & =\sum_{m=0}^{k-1} \frac{1}{m !} \frac{\partial^{m} u_{f}}{\partial t^{m}}\left(x^{\prime}, x_{n}, t\right)(y-t)^{m} \\
& -\frac{1}{k-1 !} \int_{y}^{t}(y-s)^{k-1} \frac{\partial^{k} u_{f}}{\partial s^{k}}\left(x^{\prime}, x_{n}, s\right) d s .
\end{aligned}
$$

For $h \in \mathbb{R}^{n-1}$ let us perform the finite difference operator of order $k$ and step $h$ in the variable $x^{\prime}$, then

$$
\begin{aligned}
\Delta_{h}^{k} u_{f}\left(x^{\prime}, x_{n}, y\right) & =\sum_{m=0}^{k-1} \frac{1}{m !} \Delta_{h}^{k} \frac{\partial^{m} u_{f}}{\partial t^{m}}\left(x^{\prime}, x_{n}, t\right)(y-t)^{m} \\
& -\frac{1}{k-1 !} \int_{y}^{t}(y-s)^{k-1} \Delta_{h}^{k} \frac{\partial^{k} u_{f}}{\partial s^{k}}\left(x^{\prime}, x_{n}, s\right) d s .
\end{aligned}
$$

By Lemma 3.13 applied to $\frac{\partial^{m} u_{f}}{\partial t^{m}}\left(x^{\prime}, x_{n}, t\right)$ as a function of $x^{\prime}$ for $m=0, \cdots, k-1$, where the order of derivation $\nu$ belongs to $\mathbb{N}_{0}^{n-1}$, we obtain

$$
\begin{aligned}
\Delta_{h}^{k} u_{f}\left(x^{\prime}, x_{n}, y\right) & =\sum_{m=0}^{k-1} \frac{1}{m !} \int_{\mathbb{R}} \sum_{|\nu|=k} \frac{k !}{\nu !}\left(D^{\nu} \frac{\partial^{m} u_{f}}{\partial t^{m}}\right)\left(x^{\prime}+\xi h, x_{n}, t\right) h^{\nu} M_{k}(\xi) d \xi(y-t)^{m} \\
& -\frac{1}{k-1 !} \int_{y}^{t}(y-s)^{k-1} \Delta_{h}^{k} \frac{\partial^{k} u_{f}}{\partial s^{k}}\left(x^{\prime}, x_{n}, s\right) d s .
\end{aligned}
$$

Applying Minkowski's integral inequality and noting that $\int_{\mathbb{R}} M_{k}(\xi) d \xi=1$ and that $\left\|\Delta_{h}^{k} \frac{\partial^{k} u_{f}}{\partial s^{k}}\left(\cdot, x_{n}, s\right)\right\|_{L^{p}\left(\mathbb{R}^{n-1}\right)} \leq 2^{k}\left\|\frac{\partial^{k} u_{f}}{\partial s^{k}}\left(\cdot, x_{n}, s\right)\right\|_{L^{p}\left(\mathbb{R}^{n-1}\right)}$ it turns out that

$$
\begin{aligned}
\left\|\Delta_{h}^{k} u_{f}\left(\cdot, x_{n}, y\right)\right\|_{L^{p}\left(\mathbb{R}^{n-1}\right)} & \leq \sum_{m=0}^{k-1} \frac{1}{m !} \sum_{|\nu|=k} \frac{k !}{\nu !}\left\|D^{\nu} \frac{\partial^{m} u_{f}}{\partial t^{m}}\left(\cdot, x_{n}, t\right)\right\|_{L^{p}\left(\mathbb{R}^{n-1}\right)}|h|^{k}(t-y)^{m} \\
& +\frac{2^{k}}{k-1 !} \int_{y}^{t}(s-y)^{k-1}\left\|\frac{\partial^{k} u_{f}}{\partial s^{k}}\left(\cdot, x_{n}, s\right)\right\|_{L^{p}\left(\mathbb{R}^{n-1}\right)} d s .
\end{aligned}
$$


Since by Lemma 3.14

$$
\Delta_{h}^{k} u_{f}\left(x^{\prime}, x_{n}, y\right) \rightarrow \Delta_{h}^{k} f\left(x^{\prime}, x_{n}\right)
$$

in $L^{p}\left(\mathbb{R}^{n-1}\right)$ as $y \rightarrow 0$ then

$$
\begin{aligned}
\left\|\Delta_{h}^{k} f\left(\cdot, x_{n}\right)\right\|_{L^{p}\left(\mathbb{R}^{n-1}\right)} & \leq \sum_{m=0}^{k-1} \frac{1}{m !} \sum_{|\nu|=k} \frac{k !}{\nu !}\left\|D^{\nu} \frac{\partial^{m} u_{f}}{\partial t^{m}}\left(\cdot, x_{n}, t\right)\right\|_{L^{p}\left(\mathbb{R}^{n-1}\right)}|h|^{k} t^{m} \\
& +\frac{2^{k}}{k-1 !} \int_{0}^{t} s^{k-1}\left\|\frac{\partial^{k} u_{f}}{\partial s^{k}}\left(\cdot, x_{n}, s\right)\right\|_{L^{p}\left(\mathbb{R}^{n-1}\right)} d s .
\end{aligned}
$$

Taking supremun for $|h| \leq t$, multiplying by $t^{-\alpha}$ and integrating in $(0, \infty)$ with respect to $\frac{d t}{t}$ we have

$$
\begin{aligned}
\int_{0}^{\infty} t^{-\alpha} \omega_{k}\left(f\left(\cdot, x_{n}\right), t\right)_{p} \frac{d t}{t} \\
\leq \sum_{m=0}^{k-1} \frac{1}{m !} \sum_{|\nu|=k} \frac{k !}{\nu !} \int_{0}^{\infty}\left\|t^{m+k-\alpha} D^{\nu} \frac{\partial^{m} u_{f}}{\partial t^{m}}\left(\cdot, x_{n}, t\right)\right\|_{L^{p}\left(\mathbb{R}^{n-1}\right)} \frac{d t}{t} \\
+\frac{2^{k}}{k-1 !} \int_{0}^{\infty} t^{-\alpha} \int_{0}^{t} s^{k-1}\left\|\frac{\partial^{k} u_{f}}{\partial s^{k}}\left(\cdot, x_{n}, s\right)\right\|_{L^{p}\left(\mathbb{R}^{n-1}\right)} d s \frac{d t}{t}
\end{aligned}
$$

Applying Hardy's inequality to the second term on the right hand side, integrating in $(-r, r)$ with respect to $d x_{n}$ and then applying Hölder's inequality, we get

$$
\begin{aligned}
\int_{-r}^{r} \int_{0}^{\infty} t^{-\alpha} \omega_{k}\left(f\left(\cdot, x_{n}\right), t\right)_{p} \frac{d t}{t} d x_{n} \\
\leq \sum_{m=0}^{k-1} \frac{1}{m !} \sum_{|\nu|=k} \frac{k !}{\nu !} \int_{0}^{\infty}(2 r)^{1 / p^{\prime}}\left\|t^{m+k-\alpha} D^{\nu} \frac{\partial^{m} u_{f}}{\partial t^{m}}(\cdot, t)\right\|_{L^{p}\left(\mathbb{R}^{n}\right)} \frac{d t}{t} \\
+\frac{2^{k}}{k-1 ! \alpha} \int_{0}^{\infty}(2 r)^{1 / p^{\prime}}\left\|t^{k-\alpha} \frac{\partial^{k} u_{f}}{\partial t^{k}}(\cdot, t)\right\|_{L^{p}\left(\mathbb{R}^{n}\right) \frac{d t}{t}}
\end{aligned}
$$

Using that, for $|\nu|=k$, we have (see Taibleson [11, Lemma 4 and Theorem 1])

$$
\begin{aligned}
\int_{0}^{\infty}\left\|t^{m+k-\alpha} D^{\nu} \frac{\partial^{m} u_{f}}{\partial t^{m}}(\cdot, t)\right\|_{L^{p}\left(\mathbb{R}^{n}\right) \frac{d t}{t}} & \leq c \int_{0}^{\infty}\left\|t^{k-\alpha} D^{\nu} u_{f}(\cdot, t)\right\|_{L^{p}\left(\mathbb{R}^{n}\right)} \frac{d t}{t} \\
& \leq c \int_{0}^{\infty}\left\|t^{k-\alpha} \frac{\partial^{k} u_{f}}{\partial t^{k}}(\cdot, t)\right\|_{L^{p}\left(\mathbb{R}^{n}\right)} \frac{d t}{t}
\end{aligned}
$$

then

$$
\int_{-r}^{r} \int_{0}^{\infty} t^{-\alpha} \omega_{k}\left(f\left(\cdot, x_{n}\right), t\right)_{p} \frac{d t}{t} d x_{n} \leq c r^{1 / p^{\prime}} \int_{0}^{\infty}\left\|t^{k-\alpha} \frac{\partial^{k} u_{f}}{\partial t^{k}}(\cdot, t)\right\|_{L^{p}\left(\mathbb{R}^{n}\right)} \frac{d t}{t} .
$$

This last inequality and the fact that $\int_{-r}^{r}\left\|f\left(\cdot, x_{n}\right)\right\|_{L^{p}\left(\mathbb{R}^{n-1}\right)} d x_{n}$ is less than or equal to $(2 r)^{1 / p^{\prime}}\|f\|_{L^{p}\left(\mathbb{R}^{n}\right)}$ gives the desired result. 
Proof of Lemma 3.13. We follow Bennett-Sharpley [1]. We first prove the lemma for $n=1$ by induction on $k$. Noting by $f^{\prime}$ the first derivative of $f$, we have for $k=1$,

$$
\begin{aligned}
\int_{\mathbb{R}} f^{\prime}(x+\xi h) h M_{1}(\xi) d \xi & =\int_{0}^{1} f^{\prime}(x+\xi h) h d \xi \\
& =f(x+h)-f(x) \\
& =\Delta_{h}^{1} f(x) .
\end{aligned}
$$

Assume the formula is true for $k=1, \cdots, m$ and let us prove it for $k=m+1$. We note by $f^{(k)}$ the derivative of $f$ of order $k$. Applying $\Delta_{h}^{1}$ to both sides of the induction hypothesis for $k=m, \Delta_{h}^{m} f(x)=\int_{\mathbb{R}} f^{(m)}(x+\xi h) h^{m} M_{m}(\xi) d \xi$, and using the induction hypothesis for $k=1$ applied to $f^{(m)}$ we obtain

$$
\begin{aligned}
h^{-(m+1)} \Delta_{h}^{m+1} f(x) & =\int_{\mathbb{R}} h^{-1}\left(\Delta_{h}^{1} f^{(m)}\right)(x+\xi h) M_{m}(\xi) d \xi \\
& =\int_{\mathbb{R}} h^{-1}\left(\int_{\mathbb{R}} f^{(m+1)}(x+\xi h+\tau h) h M_{1}(\tau) d \tau\right) M_{m}(\xi) d \xi \\
& =\int_{\mathbb{R}}\left(\int_{\mathbb{R}} f^{(m+1)}(x+u h) M_{1}(u-\xi) d u\right) M_{m}(\xi) d \xi \\
& =\int_{\mathbb{R}} f^{(m+1)}(x+u h)\left(\int_{\mathbb{R}} M_{1}(u-\xi) M_{m}(\xi) d \xi\right) d u \\
& =\int_{\mathbb{R}} f^{(m+1)}(x+u h) M_{m+1}(u) d u .
\end{aligned}
$$

In the next to the last equality we have used Fubini's Theorem since the integrals converge absolutely.

Assume now $n \geq 2$. Let $g(t)=f\left(x+t \frac{h}{|h|}\right), t \in \mathbb{R}$. Observe that $\Delta_{|h|}^{k} g(t)=$ $\left(\Delta_{h}^{k} f\right)\left(x+t \frac{h}{|h|}\right)$ and that $g^{(k)}(\xi|h|)=\sum_{|\nu|=k} \frac{k !}{\nu !}\left(D^{\nu} f\right)(x+\xi h)\left(\frac{h}{|h|}\right)^{\nu}$. Applying the thesis of the lemma for $n=1$ to $g$ in $t=0$ we obtain

$$
\begin{aligned}
\Delta_{h}^{k} f(x)=\Delta_{|h|}^{k} g(0) & =\int_{\mathbb{R}} g^{(k)}(\xi|h|)|h|^{k} M_{k}(\xi) d \xi \\
& =\int_{\mathbb{R}} \sum_{|\nu|=k} \frac{k !}{\nu !}\left(D^{\nu} f\right)(x+\xi h)\left(\frac{h}{|h|}\right)^{\nu}|h|^{k} M_{k}(\xi) d \xi \\
& =\int_{\mathbb{R}} \sum_{|\nu|=k} \frac{k !}{\nu !}\left(D^{\nu} f\right)(x+\xi h) h^{\nu} M_{k}(\xi) d \xi .
\end{aligned}
$$

Proof of Lemma 3.14. Let $f \in \mathcal{C}_{0}^{\infty}\left(\mathbb{R}^{n}\right)$. Observe that $u_{f}(x, y) \rightarrow f(x)$ uniformly in $\mathbb{R}^{n}$ as $y \rightarrow 0$, so in particular $u_{f}\left(x^{\prime}, x_{n}, y\right) \rightarrow f\left(x^{\prime}, x_{n}\right)$ in $L^{\infty}\left(\mathbb{R}^{n-1}\right)$ as $y \rightarrow 0$ for every $x_{n} \in \mathbb{R}$. So we may assume $1 \leq p<\infty$. We follow some ideas of Taibleson [11, Theorem 12]. 
Let $j \in \mathbb{N}_{0}$. Then

$$
\begin{aligned}
\int_{\mathbb{R}^{n-1}}\left|\frac{\partial^{j} u_{f}}{\partial y^{j}}\left(x^{\prime}, x_{n}, y\right)\right|^{p} d x^{\prime}=\int_{\mathbb{R}^{n-1}}\left|\frac{\partial^{j} u_{f}}{\partial y^{j}}\left(x^{\prime}, x_{n}, y / 2\right) * P_{y / 2}\left(x^{\prime}, x_{n}\right)\right|^{p} d x^{\prime} \\
\quad \leq \int_{x^{\prime} \in \mathbb{R}^{n-1}}\left(\int_{z \in \mathbb{R}^{n}}\left|\frac{\partial^{j} u_{f}}{\partial y^{j}}(z, y / 2)\right| P_{y / 2}\left(x^{\prime}-z^{\prime}, x_{n}-z_{n}\right) d z\right)^{p} d x^{\prime} \\
\quad \leq \int_{x^{\prime} \in \mathbb{R}^{n-1}} \int_{z \in \mathbb{R}^{n}}\left|\frac{\partial^{j} u_{f}}{\partial y^{j}}(z, y / 2)\right|^{p} P_{y / 2}\left(x^{\prime}-z^{\prime}, x_{n}-z_{n}\right) d z d x^{\prime} \\
\leq c y^{-1} \int_{z \in \mathbb{R}^{n}}\left|\frac{\partial^{j} u_{f}}{\partial y^{j}}(z, y / 2)\right|^{p} d z .
\end{aligned}
$$

In particular, $u_{f}\left(\cdot, x_{n}, y\right) \in L^{p}\left(\mathbb{R}^{n-1}\right)$ and

$$
\left\|\frac{\partial u_{f}}{\partial y}\left(\cdot, x_{n}, y\right)\right\|_{L^{p}\left(\mathbb{R}^{n-1}\right)} \leq c y^{-1 / p}\left\|\frac{\partial u_{f}}{\partial y}(\cdot, y / 2)\right\|_{L^{p}\left(\mathbb{R}^{n}\right)} .
$$

Observe that if $0<\gamma<1 / p^{\prime}$ then

$$
\left\|\frac{\partial u_{f}}{\partial y}(\cdot, y)\right\|_{L^{p}\left(\mathbb{R}^{n}\right)} \leq c y^{\gamma-1 / p^{\prime}} .
$$

In fact, taking into account that $\left\|\frac{\partial u_{f}}{\partial y}(\cdot, y)\right\|_{L^{p}\left(\mathbb{R}^{n}\right)}$ is a non increasing function of $y$ for $y \in(0,+\infty)$ (Stein [7, page 154, Lemma 6 ]) we have

$$
\begin{aligned}
\left(1 / p^{\prime}-\gamma\right)^{-1} y^{1 / p^{\prime}-\gamma}\left\|\frac{\partial u_{f}}{\partial y}(\cdot, y)\right\|_{L^{p}\left(\mathbb{R}^{n}\right)} & =\int_{0}^{y} t^{1 / p^{\prime}-\gamma}\left\|\frac{\partial u_{f}}{\partial y}(\cdot, y)\right\|_{L^{p}\left(\mathbb{R}^{n}\right) \frac{d t}{t}}^{y} \\
& \leq \int_{0}^{y} t^{1 / p^{\prime}-\gamma}\left\|\frac{\partial u_{f}}{\partial t}(\cdot, t)\right\|_{L^{p}\left(\mathbb{R}^{n}\right)} \frac{d t}{t} \\
& \leq \int_{0}^{\infty} t^{1 / p^{\prime}-\gamma}\left\|\frac{\partial u_{f}}{\partial t}(\cdot, t)\right\|_{L^{p}\left(\mathbb{R}^{n}\right)} \frac{d t}{t} .
\end{aligned}
$$

Since $f \in \mathcal{C}_{0}^{\infty}\left(\mathbb{R}^{n}\right) \subset B_{p}^{1-1 / p^{\prime}+\gamma, 1}\left(\mathbb{R}^{n}\right)$ (Lema 2.11) the last integral is finite and then we have (3.16).

From (3.15) and (3.16) we obtain $\left\|\frac{\partial u_{f}}{\partial y}\left(\cdot, x_{n}, y\right)\right\|_{L^{p}\left(\mathbb{R}^{n-1}\right)} \leq c y^{\gamma-1}$. So, if $y^{\prime}<y$,

$$
\begin{aligned}
\left\|u_{f}\left(\cdot, x_{n}, y\right)-u_{f}\left(\cdot, x_{n}, y^{\prime}\right)\right\|_{L^{p}\left(\mathbb{R}^{n-1}\right)} & \leq \int_{y^{\prime}}^{y}\left\|\frac{\partial u_{f}}{\partial t}\left(\cdot, x_{n}, t\right)\right\|_{L^{p}\left(\mathbb{R}^{n-1}\right)} d t \\
& \leq c \int_{y^{\prime}}^{y} t^{\gamma-1} d t .
\end{aligned}
$$

We then have that $u_{f}\left(x^{\prime}, x_{n}, y\right)$ converges in $L^{p}\left(\mathbb{R}^{n-1}\right)$ to a function $g_{x_{n}}\left(x^{\prime}\right) \in$ $L^{p}\left(\mathbb{R}^{n-1}\right)$ as $y \rightarrow 0$. Since $u_{f}(x, y) \rightarrow f(x)$ uniformly in $\mathbb{R}^{n}$ as $y \rightarrow 0$, it must be $g_{x_{n}}\left(x^{\prime}\right)=f\left(x^{\prime}, x_{n}\right)$ and we obtain the desired result. 


\section{Proofs of 1.2.a and 1.3. $a$ (positive results)}

Since the proof of Theorem 1.4 follows that of Stokolos [9, Lemma1] which makes use of the non-increasing rearrangement of a function, we give its definition here and we state a property (equality (4.17)) which will be useful in the proofs of both Theorem 1.4 and Lemma 4.18 below.

Suppose $f$ is a measurable function in $\mathbb{R}^{n}$. The non-increasing rearrangement of $f$ is the function $f^{*}$ defined in $[0,+\infty)$ by

$$
f^{*}(t)=\inf \left\{\lambda \geq 0: m_{f}(\lambda) \leq t\right\} \quad t \geq 0,
$$

where $m_{f}$ is the distribution function of $f$, that is, $m_{f}(\lambda)=\mid\left\{x \in \mathbb{R}^{n}:|f(x)|>\right.$ $\lambda\} \mid$.

Let $t>0$, then

$$
\int_{0}^{t} f^{*}(s) d s=\sup _{|E|=t, E \subset \mathbb{R}^{n}} \int_{E}|f(x)| d x
$$

See for example Bennett-Sharpley [1, page 53, Propotition 3.3 ].

For the proof of Theorem 1.4 we also need the following estimate of rearrangements in terms of Besov norms.

Lemma 4.18. For $1 \leq p<\infty$ we have

$$
\frac{1}{t} \int_{0}^{t} f^{*}(s) d s \leq c\|f\|_{B_{p}^{n / p, 1}\left(\mathbb{R}^{n}\right)}
$$

for all $t>0$. Here $c$ is a constant independent of $f$ and $t$.

Proof of Theorem 1.4. Fix $1 \leq p<\infty$ and $r>0$. By part $a$ ) of Lemma 2.11 and using standard arguments it is enough to prove inequality (1.5) for $f \in \mathcal{C}_{0}^{\infty}\left(\mathbb{R}^{n}\right)$ with $\operatorname{supp}(f) \subset B(0, r)$. We follow Stokolos [9, Lemma 1] to get the proof of the theorem.

Let $f \in \mathcal{C}_{0}^{\infty}\left(\mathbb{R}^{n}\right)$ with $\operatorname{supp}(f) \subset B(0, r)$ and $x=\left(x^{\prime}, x_{n}\right) \in R \in \mathcal{B}$. It can be shown that there exists a measurable set $\bar{R}$, containing $R$, whose measure and diameter are comparable to those of $R$, whose projection onto a coordinate axis, $y_{n}$ for instance, is an interval $I$ and the section of $\bar{R}$ by the hyperplane $y_{n}=t$, denoted by $\bar{R}_{t}$ (i.e. $\bar{R}_{t}=\left\{y^{\prime} \in \mathbb{R}^{n-1}:\left(y^{\prime}, t\right) \in \bar{R}\right\}$ ), has constant measure for $t \in I$. We denote by $f^{*}\left(t, y_{n}\right)$ the non-increasing rearrangement of $f\left(y^{\prime}, y_{n}\right)$ for each fixed $y_{n}$ as a function of $y^{\prime}$. Then, using (4.17) and Lemma 4.18 we have

$$
\begin{aligned}
\frac{1}{|R|} \int_{R}|f(y)| d y & \leq c \frac{1}{|\bar{R}|} \int_{\bar{R}}|f(y)| d y \\
& =c \frac{1}{|I|} \int_{I} \frac{1}{\left|\bar{R}_{y_{n}}\right|} \int_{\bar{R}_{y_{n}}}\left|f\left(y^{\prime}, y_{n}\right)\right| d y^{\prime} d y_{n} \\
& \leq c \frac{1}{|I|} \int_{I} \frac{1}{\left|\bar{R}_{y_{n}}\right|} \int_{0}^{\left|\bar{R}_{y_{n}}\right|} f^{*}\left(t, y_{n}\right) d t d y_{n} \\
& \leq c \frac{1}{|I|} \int_{I}\left\|f\left(\cdot, y_{n}\right)\right\|_{B_{p}^{(n-1) / p, 1}\left(\mathbb{R}^{n-1}\right)} d y_{n}
\end{aligned}
$$


Then, if $\mathcal{M}_{h l}$ denotes the Hardy-Littlewood maximal operator on $\mathbb{R}$, it turns out that

$$
\mathcal{M} f(x) \leq c \mathcal{M}_{h l}\left(\|f(\cdot, \cdot)\|_{B_{p}^{(n-1) / p, 1}\left(\mathbb{R}^{n-1}\right)}\right)\left(x_{n}\right),
$$

and since the sets in $\mathcal{B}$ have diameter smaller than $1, \operatorname{supp}(f) \subset B(0, r)$ and $\mathcal{M}_{h l}$ is of weak type $(1,1)$, it follows that

$$
\begin{aligned}
\left|\left\{x \in \mathbb{R}^{n}: \mathcal{M} f(x)>\lambda\right\}\right| & =|\{x \in B(0, r+1): \mathcal{M} f(x)>\lambda\}| \\
& \leq c \frac{(r+1)^{n-1}}{\lambda} \int_{-r}^{r}\left\|f\left(\cdot, x_{n}\right)\right\|_{B_{p}^{(n-1) / p, 1}\left(\mathbb{R}^{n-1}\right)} d x_{n} .
\end{aligned}
$$

Taking into account the result of Theorem 3.12, we complete the proof of the theorem.

Proof of part a) of Theorem 1.2. By part b) of Lemma 2.11 it is enough to prove the theorem for functions of compact support. Let $f \in B_{p}^{(n-1) / p, 1}\left(\mathbb{R}^{n}\right)$, $\operatorname{supp}(f) \subset B(0, r), r>0$ and

$$
\begin{aligned}
\Gamma f(x) & =\limsup _{R \rightarrow x} \frac{1}{|R|} \int_{R}|f(y)-f(x)| d y \\
& =\inf _{\delta>0} \sup \left\{\frac{1}{|R|} \int_{R}|f(y)-f(x)| d y, x \in R \in \mathcal{B}, \operatorname{diam}(R)<\delta\right\} .
\end{aligned}
$$

Set $t>0$ and $E_{t}(f)=\left\{x \in \mathbb{R}^{n}: \Gamma f(x)>t\right\}$. We are done if we prove that $\left|E_{t}(f)\right|_{e}=0$ for every $t>0$, where $|.|_{e}$ denotes outer measure.

By part $a$ ) of Lemma 2.11, given $\epsilon>0$ there exists $g \in \mathcal{C}_{0}^{\infty}\left(\mathbb{R}^{n}\right)$ such that $\operatorname{supp}(g) \subset B(0,2 r)$ and $\|f-g\|_{B_{p}^{(n-1) / p, 1}\left(\mathbb{R}^{n}\right)}<\epsilon$. We have

$$
\begin{aligned}
\Gamma f(x) & \leq \Gamma(f-g)(x)+\Gamma g(x) \\
& \leq \mathcal{M}(f-g)(x)+|f(x)-g(x)|+\Gamma g(x) \\
& =\mathcal{M}(f-g)(x)+|f(x)-g(x)| .
\end{aligned}
$$

So, using Theorem 1.4,

$$
\begin{aligned}
\left|E_{t}(f)\right|_{e} & \leq\left|\left\{x \in \mathbb{R}^{n}: \mathcal{M}(f-g)(x)>\frac{t}{2}\right\}\right|+\left|\left\{x \in \mathbb{R}^{n}:|f(x)-g(x)|>\frac{t}{2}\right\}\right| \\
& \leq(c / t)(2 r+1)^{n-1}(2 r)^{1 / p^{\prime}}\|f-g\|_{B_{p}^{(n-1) / p, 1}\left(\mathbb{R}^{n}\right)}+(2 / t)^{p}\|f-g\|_{L^{p}\left(\mathbb{R}^{n}\right)}^{p} \\
& \leq(c / t)(2 r+1)^{n-1}(2 r)^{1 / p^{\prime}} \epsilon+(2 / t)^{p} \epsilon^{p} .
\end{aligned}
$$

Since $\epsilon$ is arbitrary, this ends the proof of the theorem.

Proof of part a) of Corollary 1.3. It is a direct consequence of part a) of Theorem 1.2 and Proposition 2.10. In fact, we have $B_{p}^{\alpha, q}\left(\mathbb{R}^{n}\right) \subset B_{p}^{(n-1) / p, 1}\left(\mathbb{R}^{n}\right)$ if $\alpha>\frac{n-1}{p}, 1 \leq q \leq \infty$ and $1 \leq p<\infty$.

Proof of Lemma 4.18. We will prove the lemma using a result which is essentially Calderón's representation formula for tempered distributions. More 
precisely, it can be shown that for $\mu$ a finite Borel measure satisfying the standard Tauberian condition (for all $\xi \in \mathbb{R}^{n}, \xi \neq 0$, there exists $s>0$ such that $\hat{\mu}(s \xi) \neq 0)$ there exists $\eta \in \mathcal{S}\left(\mathbb{R}^{n}\right)$ such that $\operatorname{supp}(\hat{\eta})$ is contained in an annulus and $\int_{0}^{\infty} \hat{\mu}(s \xi) \hat{\eta}(s \xi) \frac{d s}{s}=1$ for $\xi \neq 0$ (Heideman $[5]$ ).

Fix $1 \leq p<\infty$. We assume $f \in B_{p}^{n / p, 1}\left(\mathbb{R}^{n}\right)$ since otherwise there is nothing to prove. Consider in $B_{p}^{n / p, 1}\left(\mathbb{R}^{n}\right)$ the norm $(2.9)$ and let $\eta \in \mathcal{S}\left(\mathbb{R}^{n}\right)$ be associated to $\mu=\varphi(x) d x$ according to Heideman's result above. We then define

$$
\hat{\psi}(\xi)=\left\{\begin{array}{cc}
\int_{1}^{\infty} \hat{\varphi}(s \xi) \hat{\eta}(s \xi) \frac{d s}{s}, & \xi \neq 0, \\
1, & \xi=0 .
\end{array}\right.
$$

Observe that $\hat{\psi} \in \mathcal{C}_{0}^{\infty}\left(\mathbb{R}^{n}\right)$ and $\hat{\psi}=1$ in a neighborhood of the origin. In fact, if $\operatorname{supp}(\hat{\eta}) \subset\{\xi: 0<a<|\xi|<b\}$, then $\hat{\psi}(\xi)=1$ if $|\xi|<a$ and $\hat{\psi}(\xi)=0$ if $|\xi|>b$. It is now clear that $\hat{\psi} \in \mathcal{C}_{0}^{\infty}\left(\mathbb{R}^{n}\right)$. Then $\psi \in \mathcal{S}\left(\mathbb{R}^{n}\right)$ and $\int_{\mathbb{R}^{n}} \psi(x) d x=1$. So that $\psi$ is a good approximation to the identity.

Set $I_{\epsilon, a}(x)=\int_{\epsilon}^{a}\left(\varphi_{s} * \eta_{s}\right)(x) \frac{d s}{s}, \epsilon<a$. The function $I_{\epsilon, a} \in L^{1}\left(\mathbb{R}^{n}\right)$ since

$$
\begin{aligned}
\int_{\mathbb{R}^{n}}\left|I_{\epsilon, a}(x)\right| d x & \leq \int_{\epsilon}^{a} \int_{\mathbb{R}^{n}}\left|\left(\varphi_{s} * \eta_{s}\right)(x)\right| d x \frac{d s}{s} \\
& \leq\|\varphi\|_{L^{1}\left(\mathbb{R}^{n}\right)}\|\eta\|_{L^{1}\left(\mathbb{R}^{n}\right)} \log \left(\frac{a}{\epsilon}\right) .
\end{aligned}
$$

By Fubini's theorem,

$$
\begin{aligned}
\hat{I_{\epsilon, a}}(\xi) & =\int_{\mathbb{R}^{n}} \int_{\epsilon}^{a}\left(\varphi_{s} * \eta_{s}\right)(x) e^{-i x \xi} \frac{d s}{s} d x \\
& =\int_{\epsilon}^{a} \int_{\mathbb{R}^{n}}\left(\varphi_{s} * \eta_{s}\right)(x) e^{-i x \xi} d x \frac{d s}{s} \\
& =\int_{\epsilon}^{a}\left(\varphi_{s} * \eta_{s}\right)(\xi) \frac{d s}{s} \\
& =\int_{\epsilon}^{a} \hat{\varphi}(s \xi) \hat{\eta}(s \xi) \frac{d s}{s} \\
& =\hat{\psi}(\epsilon \xi)-\hat{\psi}(a \xi) \\
& =\hat{\psi}_{\epsilon}(\xi)-\hat{\psi}_{a}(\xi) .
\end{aligned}
$$

So $I_{\epsilon, a} \in \mathcal{S}\left(\mathbb{R}^{n}\right)$ and $I_{\epsilon, a}=\psi_{\epsilon}-\psi_{a}$. Then, since convolution with $f$ commutes with the integral, we arrive at

$$
\left(\psi_{\epsilon} * f\right)(x)-\left(\psi_{a} * f\right)(x)=\left(I_{\epsilon, a} * f\right)(x)=\int_{\epsilon}^{a}\left(\varphi_{s} * \eta_{s} * f\right)(x) \frac{d s}{s} .
$$

Applying Minkowski's integral inequality and Young's inequality we have

$$
\left\|\psi_{\epsilon} * f-\psi_{a} * f\right\|_{L^{p}\left(\mathbb{R}^{n}\right)} \leq\|\eta\|_{L^{1}\left(\mathbb{R}^{n}\right)} \int_{\epsilon}^{a}\left\|\varphi_{s} * f\right\|_{L^{p}\left(\mathbb{R}^{n}\right)} \frac{d s}{s}
$$


and since $\psi_{\epsilon} * f \rightarrow f$ in $L^{p}\left(\mathbb{R}^{n}\right)$ as $\epsilon \rightarrow 0$, it turns out that

$$
\left\|f-\psi_{a} * f\right\|_{L^{p}\left(\mathbb{R}^{n}\right)} \leq\|\eta\|_{L^{1}\left(\mathbb{R}^{n}\right)} \int_{0}^{a}\left\|\varphi_{s} * f\right\|_{L^{p}\left(\mathbb{R}^{n}\right)} \frac{d s}{s} .
$$

On the other hand, taking $a=1\left(\psi_{a}=\psi\right)$ in (4.19) and applying Hölder's inequality,

$$
\begin{aligned}
\left|\left(\psi_{\epsilon} * f\right)(x)\right| & \leq|(\psi * f)(x)|+\int_{\epsilon}^{1}\left|\left(\varphi_{s} * \eta_{s} * f\right)(x)\right| \frac{d s}{s} \\
& \leq\|\psi\|_{L^{p^{\prime}}\left(\mathbb{R}^{n}\right)}\|f\|_{L^{p}\left(\mathbb{R}^{n}\right)}+\int_{\epsilon}^{1}\left\|\eta_{s}\right\|_{L^{p^{\prime}\left(\mathbb{R}^{n}\right)}}\left\|\varphi_{s} * f\right\|_{L^{p}\left(\mathbb{R}^{n}\right)} \frac{d s}{s} \\
& =\|\psi\|_{L^{p^{\prime}\left(\mathbb{R}^{n}\right)}}\|f\|_{L^{p}\left(\mathbb{R}^{n}\right)}+\|\eta\|_{L^{p^{\prime}}\left(\mathbb{R}^{n}\right)} \int_{\epsilon}^{1} s^{-n / p}\left\|\varphi_{s} * f\right\|_{L^{p}\left(\mathbb{R}^{n}\right)} \frac{d s}{s} \\
& \leq c\left(\|f\|_{L^{p}\left(\mathbb{R}^{n}\right)}+\int_{\epsilon}^{1} s^{-n / p}\left\|\varphi_{s} * f\right\|_{\left.L^{p}\left(\mathbb{R}^{n}\right) \frac{d s}{s}\right) .}\right.
\end{aligned}
$$

Then, using (4.17), Hölder's inequality, (4.20) and (4.21) we have, for $a<1$,

$$
\begin{aligned}
& \frac{1}{t} \int_{0}^{t} f^{*}(s) d s=\frac{1}{t} \sup _{E \subset \mathbb{R}^{n},|E|=t} \int_{E}|f(x)| d x \\
& \quad \leq \frac{1}{t} \sup _{E \subset \mathbb{R}^{n},|E|=t} \int_{E}\left|f(x)-\left(\psi_{a} * f\right)(x)\right| d x+\left\|\psi_{a} * f\right\|_{L^{\infty}\left(\mathbb{R}^{n}\right)} \\
& \leq \frac{1}{t^{1 / p}}\left\|f-\psi_{a} * f\right\|_{L^{p}\left(\mathbb{R}^{n}\right)}+\left\|\psi_{a} * f\right\|_{L^{\infty}\left(\mathbb{R}^{n}\right)} \\
& \leq \frac{\|\eta\|_{L^{1}\left(\mathbb{R}^{n}\right)}}{t^{1 / p}} \int_{0}^{a}\left\|\varphi_{s} * f\right\|_{L^{p}\left(\mathbb{R}^{n}\right)} \frac{d s}{s}+c\left(\|f\|_{L^{p}\left(\mathbb{R}^{n}\right)}\right. \\
& \left.\quad+\int_{a}^{1} s^{-n / p}\left\|\varphi_{s} * f\right\|_{L^{p}\left(\mathbb{R}^{n}\right)} \frac{d s}{s}\right) .
\end{aligned}
$$

If $t<1$ and $a=t^{1 / n}$ we obtain

$$
\frac{1}{t^{1 / p}} \int_{0}^{t^{1 / n}}\left\|\varphi_{s} * f\right\|_{L^{p}\left(\mathbb{R}^{n}\right)} \frac{d s}{s} \leq \int_{0}^{t^{1 / n}} s^{-n / p}\left\|\varphi_{s} * f\right\|_{L^{p}\left(\mathbb{R}^{n}\right)} \frac{d s}{s}
$$

and then

$$
\frac{1}{t} \int_{0}^{t} f^{*}(s) d s \leq c\|f\|_{B_{p}^{n / p, 1}\left(\mathbb{R}^{n}\right)}, \quad t<1
$$

If $t \geq 1$ then

$$
\begin{aligned}
\frac{1}{t} \int_{0}^{t} f^{*}(s) d s & \leq \frac{1}{t^{1 / p}}\left(\int_{0}^{t} f^{*}(s)^{p} d s\right)^{\frac{1}{p}} \\
& \leq\left(\int_{0}^{\infty} f^{*}(s)^{p} d s\right)^{\frac{1}{p}}=\|f\|_{L^{p}\left(\mathbb{R}^{n}\right)} \leq\|f\|_{B_{p}^{n / p, 1}\left(\mathbb{R}^{n}\right)}
\end{aligned}
$$




\section{Proofs of 1.2.b, 1.3.b and 1.6 (negative results)}

Proof of part b) of Theorem 1.2. Fix $1 \leq p<n$ and $0<\alpha<\frac{n}{p}-1$. Following Stokolos $[9,10]$, we consider in $Q_{0}=\left\{\left(y_{1}, \cdots, y_{n}\right):-\frac{1}{2}<y_{i} \leq \frac{1}{2}, i=1, \cdots, n\right\}$, $m^{n}$ disjoint equal cubes $I_{j}^{m}$ of measure $\left|I_{j}^{m}\right|=m^{-n}$. Let $Q_{j}^{m}$ be the cube concentric with $I_{j}^{m}$ of measure $\left|Q_{j}^{m}\right|=2^{-n m}$. Let $\psi$ be a non-negative function in $B_{\infty}^{(n-1) / p, \infty}\left(\mathbb{R}^{n}\right)$ with $\operatorname{supp}(\psi) \subset B(0,1)$ and $\psi \equiv 1$ in $B\left(0, \frac{1}{2}\right)$. We define $f_{j}^{m}(x)=2^{m} \psi\left(2^{m+1}\left(x-x_{j}^{m}\right)\right)$, where $x_{j}^{m}$ is the center of $Q_{j}^{m}$. Then $\operatorname{supp}\left(f_{j}^{m}\right) \subset Q_{j}^{m}$ and since, from $(2.8),\left|\Delta_{h}^{n} \psi(x)\right| \leq c|h|^{(n-1) / p}$ for every $h \in \mathbb{R}^{n}$ and $x \in \mathbb{R}^{n}$, we have

$$
\begin{aligned}
\left|\Delta_{h}^{n} f_{j}^{m}(x)\right| & =\left|\sum_{i=0}^{n}\left(\begin{array}{c}
n \\
i
\end{array}\right)(-1)^{n-i} f_{j}^{m}(x+i h)\right| \\
& =\left|2^{m} \sum_{i=0}^{n}\left(\begin{array}{c}
n \\
i
\end{array}\right)(-1)^{n-i} \psi\left(2^{m+1}\left(x+i h-x_{j}^{m}\right)\right)\right| \\
& =\left|2^{m} \Delta_{2^{m+1} h}^{n} \psi\left(2^{m+1}\left(x-x_{j}^{m}\right)\right)\right| \\
& \leq c 2^{m}\left|2^{m+1} h\right|^{\frac{n-1}{p}} \\
& =c 2^{m\left(1+\frac{n-1}{p}\right)}|h|^{\frac{n-1}{p}} .
\end{aligned}
$$

Set

$$
f(x)=\sum_{m=1}^{\infty} \sum_{j=1}^{m^{n}} f_{j}^{m}(x)
$$

for $x \in \mathbb{R}^{n}$. Clearly $f \in L^{p}\left(\mathbb{R}^{n}\right)$ since

$$
\|f\|_{L^{p}\left(\mathbb{R}^{n}\right)} \leq \sum_{m=1}^{\infty} \sum_{j=1}^{m^{n}}\left\|f_{j}^{m}\right\|_{L^{p}\left(\mathbb{R}^{n}\right)}=2^{-\frac{n}{p}}\|\psi\|_{L^{p}\left(\mathbb{R}^{n}\right)} \sum_{m=1}^{\infty} m^{n} 2^{m\left(1-\frac{n}{p}\right)}<\infty .
$$

Moreover $f \in B_{p}^{\alpha, 1}\left(\mathbb{R}^{n}\right)$. In fact, if $t>0$ and $l \in \mathbb{N}$ are such that $2^{-(l+1)} \leq t<$ $2^{-l}$ and $h \in \mathbb{R}^{n}$ with $|h|<t$, we have

$$
\begin{aligned}
\left\|\Delta_{h}^{n} f\right\|_{L^{p}\left(\mathbb{R}^{n}\right)} & \leq \sum_{m=1}^{l} \sum_{j=1}^{m^{n}}\left\|\Delta_{h}^{n} f_{j}^{m}\right\|_{L^{p}\left(c_{n} Q_{j}^{m}\right)}+2^{n} \sum_{m=l+1}^{\infty} \sum_{j=1}^{m^{n}}\left\|f_{j}^{m}\right\|_{L^{p}\left(\mathbb{R}^{n}\right)} \\
& \leq c\left(|h|^{\frac{n-1}{p}} \sum_{m=1}^{l} m^{n} 2^{m\left(1+\frac{n-1}{p}\right)} 2^{-\frac{n m}{p}}+\sum_{m=l+1}^{\infty} m^{n} 2^{m\left(1-\frac{n}{p}\right)}\right) \\
& \leq c\left(|h|^{\frac{n-1}{p}} l^{n+1} 2^{l\left(1-\frac{1}{p}\right)}+\sum_{m=l+1}^{\infty} m^{n} 2^{m\left(1-\frac{n}{p}\right)}\right) .
\end{aligned}
$$

Since $|h|<t, \sum_{m=l+1}^{\infty} m^{n} 2^{m\left(1-\frac{n}{p}\right)} \leq c(l+1)^{n+1} 2^{(l+1)\left(1-\frac{n}{p}\right)}$ for $1 \leq p<n$ and $t \geq 2^{-(l+1)}$, then both terms on the right hand side of the above inequality are 
bounded above by $c t^{\frac{n-1}{p}} l^{n+1} 2^{l\left(1-\frac{1}{p}\right)}$. We then have,

$$
\begin{aligned}
\int_{0}^{1} t^{-\alpha} \omega_{n}(f, t)_{p} \frac{d t}{t} & \leq c \sum_{l=0}^{\infty} \int_{2^{-(l+1)}}^{2^{-l}} t^{\frac{n-1}{p}-\alpha} l^{n+1} 2^{l\left(1-\frac{1}{p}\right)} \frac{d t}{t} \\
& =c \sum_{l=0}^{\infty} l^{n+1} 2^{l\left(1-\frac{1}{p}\right)}\left(2^{-l\left(\frac{n-1}{p}-\alpha\right)}-2^{-(l+1)\left(\frac{n-1}{p}-\alpha\right)}\right)<\infty
\end{aligned}
$$

since $\alpha<\frac{n}{p}-1$.

Let us now prove that

$$
\limsup _{\operatorname{diam}(R) \rightarrow 0, x \in R \in \mathcal{B}} \frac{1}{|R|} \int_{R} f(y) d y=+\infty
$$

for every $x \in Q_{0}$ by showing that if $x \in I_{j}^{m}$, there exists a convex set $K(x)$, and then a rectangular parallelepiped (de Guzmán [3, page 139, Lemma 2.2 (John's Lemma) ]), such that $x \in K(x), \operatorname{diam}(K(x)) \leq c m^{-1}$ and

$$
\frac{1}{|K(x)|} \int_{K(x)} f_{j}^{m}(y) d y \geq c m
$$

Set $x \in I_{j}^{m}$ and consider the closed segment $J$ joining $x$ with $x_{j}^{m}$. Let $B_{j}^{m}$ be the ball with center $x_{j}^{m}$ circumscribing $Q_{j}^{m}$ (that is, $B_{j}^{m}=B\left(x_{j}^{m},(\sqrt{n} / 2) 2^{-m}\right)$ ) and $K(x)=\cup_{y \in J} B\left(y,(\sqrt{n} / 2) 2^{-m}\right)$. Then $Q_{j}^{m} \subset K(x)$,

$$
\operatorname{diam}(K(x))=l(J)+\operatorname{diam}\left(B_{j}^{m}\right) \leq 2 \operatorname{diam}\left(I_{j}^{m}\right) \leq c m^{-1},
$$

and

$$
|K(x)| \leq c\left(l(J)+\operatorname{diam}\left(B_{j}^{m}\right)\right)\left(\operatorname{diam}\left(B_{j}^{m}\right)\right)^{n-1} \leq c m^{-1} 2^{-(n-1) m} .
$$

Finally, observing that $f_{j}^{m}(x)=2^{m}$ in $\frac{1}{2 \sqrt{n}} Q_{j}^{m}$, the cube concentric with $Q_{j}^{m}$ whose side measures $\frac{1}{2 \sqrt{n}}$ times the side of $Q_{j}^{m}$, we have

$$
\frac{1}{|K(x)|} \int_{K(x)} f_{j}^{m}(y) d y \geq c \frac{2^{m}\left|\frac{1}{2 \sqrt{n}} Q_{j}^{m}\right|}{2^{-(n-1) m} m^{-1}}=c m .
$$

Proof of part b) of Corollary 1.3. It is a consequence of part $b$ ) of Theorem 1.2 and Proposition 2.10 since we have $B_{p}^{\alpha, 1}\left(\mathbb{R}^{n}\right) \subset B_{p}^{\alpha, q}\left(\mathbb{R}^{n}\right), q \geq 1$.

Proof of Theorem 1.6. It is a consequence of the proof of part $b$ ) of Theorem 1.2 and Proposition 2.10. 


\section{Stokolos' basis of pliable tubes}

Theorem 1.4 is valid for more general bases. Given $i=1, \cdots, n$, let $E$ be a measurable set whose projection onto the $x_{i}$-axis is an interval $I$ and such that the section of $E$ by the hyperplane $x_{i}=t$ has constant measure for $t \in I$. We denote by $\mathcal{P}_{i}$ the differentiation basis of all such sets with diameter smaller than 1 and set $\mathcal{P}=\cup_{i=1}^{n} \mathcal{P}_{i}$. This is what Stokolos [9] calls the basis of pliable tubes. It is clear that the proof of Theorem 1.4 allows to show inequality (1.5) for the maximal operator associated to $\mathcal{P}$ instead of $\mathcal{M}$. In fact, we have used in the proof that every element of $\mathcal{B}$ is contained in an element of $\mathcal{P}$ of comparable measure and diameter. So $\mathcal{P}$ differentiates $B_{p}^{\alpha, q}\left(\mathbb{R}^{n}\right)$ with $\alpha, p$ and $q$ in the range of the positive results for $\mathcal{B}$. We can give a complete answer to the question of differentiation of integrals of Besov functions in this case.

Theorem 6.22. Let $0<\alpha<\frac{n-1}{p}, 1 \leq p<\infty$ and $1 \leq q \leq \infty$. Then $\mathcal{P}$ does not differentiate $B_{p}^{\alpha, q}\left(\mathbb{R}^{n}\right)$.

Proof. We use the following

Lemma 6.23. Let $\alpha>0,1 \leq p, q \leq \infty, m, n \in \mathbb{N}, m \leq n$. For $\phi \in B_{p}^{\alpha, q}\left(\mathbb{R}^{m}\right)$ and $g \in B_{p}^{\alpha, q}\left(\mathbb{R}^{n-m}\right)$ let

$$
f\left(x_{1}, \cdots, x_{n}\right)=\phi\left(x_{1}, \cdots, x_{m}\right) g\left(x_{m+1}, \cdots, x_{n}\right) .
$$

Then $f \in B_{p}^{\alpha, q}\left(\mathbb{R}^{n}\right)$.

The proof of Theorem 6.22 we present here is essentially that of Stokolos [9, Theorem 1]. Fix $0<\alpha<\frac{n-1}{p}, 1 \leq p<\infty$ and $1 \leq q \leq \infty$, then there exists a non-negative function $v \in B_{p}^{\alpha, q}\left(\mathbb{R}^{n-1}\right)$ such that $v \notin L^{\infty}\left(\mathbb{R}^{n-1}\right)$. Let $\left\{m_{i}\right\}_{i \in \mathbb{N}}$ be a dense subset in $\mathbb{R}^{n-1}$ and define

$$
\phi(y)=\sum_{i=1}^{\infty} 2^{-i} v\left(y+m_{i}\right), \quad y \in \mathbb{R}^{n-1} .
$$

Then $\phi \in B_{p}^{\alpha, q}\left(\mathbb{R}^{n-1}\right)$ and $\phi$ is unbounded in every neighborhood of every point of $\mathbb{R}^{n-1}$. Let $g \in B_{p}^{\alpha, q}(\mathbb{R})$ be a non-negative function such that $g=1$ in $(-1,1)$ and consider

$$
f\left(x_{1}, \cdots, x_{n}\right)=\phi\left(x_{1}, \cdots, x_{n-1}\right) g\left(x_{n}\right) .
$$

Then, by Lemma $6.23, f \in B_{p}^{\alpha, q}\left(\mathbb{R}^{n}\right)$. We will see that $\mathcal{P}$ does not differentiate the integral of $f$ by showing that for every $x=\left(x_{1}, \cdots, x_{n}\right)$ with $\left|x_{n}\right|<1$ we have

$$
\limsup _{\operatorname{diam}(E) \rightarrow 0, x \in E \in \mathcal{P}} \frac{1}{|E|} \int_{E} f(y) d y=+\infty .
$$

Fix $x=\left(x_{1}, \cdots, x_{n}\right) \in \mathbb{R}^{n}$ with $\left|x_{n}\right|<1$. Given an $n-1$ dimensional neighborhood $N$ of $\left(x_{1}, \cdots, x_{n-1}\right)$ let $Q$ be an $(n-1)$-dimensional ball contained in $N$ such that $\frac{1}{|Q|} \int_{Q} \phi\left(y^{\prime}\right) d y^{\prime}$ is great enough. Let $w=\left(w_{1}, \cdots, w_{n-1}\right)$ be the center of $Q$ and $a<b<c$ numbers such that $b-a=c-b,(a, c) \subset(-1,1)$ and 
$x_{n} \in(b, c)$. Let $J$ be the segment through $x$ starting at $\left(w_{1}, \cdots, w_{n-1}, b\right)$ and such that its projection onto the $x_{n}$-axis is the closed interval $[b, c]$. We define $E_{1}$ as the union of all balls congruent to $Q$ with center in $J$ and $E_{2}=Q \times(a, b)$. Let $E=E_{1} \cup E_{2}$, so $E \in \mathcal{P}, x \in E,|E|=2|Q|(b-a)$ and the diameter of $E$ can be made small enough taking $N$ and $c-a$ small enough; moreover we have

$$
\frac{1}{|E|} \int_{E} f(y) d y \geq \frac{1}{2|Q|(b-a)} \int_{E_{2}} f(y) d y=\frac{1}{2|Q|} \int_{Q} \phi\left(y^{\prime}\right) d y^{\prime} .
$$

Proof of Lemma 6.23. Let $\phi, g, f$ be as in the statement of the lemma. Observe that $f \in L^{p}\left(\mathbb{R}^{n}\right)$, since $\|f\|_{L^{p}\left(\mathbb{R}^{n}\right)}=\|\phi\|_{L^{p}\left(\mathbb{R}^{m}\right)}\|g\|_{L^{p}\left(\mathbb{R}^{n-m}\right)}$. Let $t>0, h \in \mathbb{R}$, $|h|<t$ and $k \in \mathbb{N}, k>\alpha$. Then, if $j=1, \cdots, m$, we have

$$
\begin{aligned}
\Delta_{h e_{j}}^{k} f\left(x_{1}, \cdots, x_{n}\right) & =\sum_{i=0}^{k}\left(\begin{array}{c}
k \\
i
\end{array}\right)(-1)^{k-i} \phi\left(x_{1}, \cdots, x_{j}+i h, \cdots, x_{m}\right) g\left(x_{m+1}, \cdots, x_{n}\right) \\
& =g\left(x_{m+1}, \cdots, x_{n}\right) \Delta_{h e_{j}}^{k} \phi\left(x_{1}, \cdots, x_{m}\right) .
\end{aligned}
$$

So, $\left\|\Delta_{h e_{j}}^{k} f\right\|_{L^{p}\left(\mathbb{R}^{n}\right)}=\|g\|_{L^{p}\left(\mathbb{R}^{n-m}\right)}\left\|\Delta_{h e_{j}}^{k} \phi\right\|_{L^{p}\left(\mathbb{R}^{m}\right)}$ and then

$$
\omega_{k}^{j}(f, t)_{p}:=\sup _{|h|<t, h \in \mathbb{R}}\left\|\Delta_{h e_{j}}^{k} f\right\|_{L^{p}\left(\mathbb{R}^{n}\right)}=\|g\|_{L^{p}\left(\mathbb{R}^{n-m}\right)} \omega_{k}^{j}(\phi, t)_{p}
$$

for $j=1, \cdots, m$. Analogously it follows that

$$
\omega_{k}^{j}(f, t)_{p}=\|\phi\|_{L^{p}\left(\mathbb{R}^{m}\right)} \omega_{k}^{j}(g, t)_{p} .
$$

if $j=m+1, \cdots, n$. Since (Triebel [12])

$$
\|f\|_{B_{p}^{\alpha, q}\left(\mathbb{R}^{n}\right)} \sim\|f\|_{L^{p}\left(\mathbb{R}^{n}\right)}+\sum_{j=1}^{n}\left(\int_{0}^{1}\left(t^{-\alpha} \omega_{k}^{j}(f, t)_{p}\right)^{q} \frac{d t}{t}\right)^{\frac{1}{q}},
$$

with the obvious changes for $q=\infty, \phi \in B_{p}^{\alpha, q}\left(\mathbb{R}^{m}\right)$ and $g \in B_{p}^{\alpha, q}\left(\mathbb{R}^{n-m}\right)$, we have that $f \in B_{p}^{\alpha, q}\left(\mathbb{R}^{n}\right)$.

\section{References}

[1] C. Bennett and R. Sharpley, Interpolation of operators, Pure and Applied Mathematics, 129. Academic Press, Inc., Boston, MA, 1988.

[2] H. Q. Bui, M. Paluszyński and M. Taibleson, A note on the Besov-Lipschitz and TriebelLizorkin spaces, Harmonic analysis and operator theory (Caracas, 1994), 95-101, Contemp. Math., 189, Amer. Math. Soc., Providence, RI, 1995.

[3] M. de Guzmán, Differentiation of integrals in $\mathbb{R}^{n}$, Lecture Notes in Mathematics, Vol. 481. Springer-Verlag, Berlin-New York, 1975.

[4] , Real variable methods in Fourier analysis, North-Holland Mathematics Studies, 46. Notas de Matemtica, 75. North-Holland Publishing Co., Amsterdam-New York, 1981.

[5] N. J. Heideman, Duality and fractional integration in Lipschitz spaces, Studia Math. 50 (1974), 65-85.

[6] O. Nikodym, Sur les ensembles accessibles, Fund. Math. 10 (1927), 116-168.

[7] E. M. Stein, Singular integrals and differentiability properties of functions, Princeton Mathematical Series, No. 30, Princeton University Press, Princeton, NJ, 1970. 
[8] _ Harmonic analysis: Real-variable methods, orthogonality, and oscillatory integrals, Princeton Mathematical Series, 43. Monographs in Harmonic Analysis, III. Princeton University Press, Princeton, NJ, 1993.

[9] A. M. Stokolos, On the differentiation of integrals by bases that do not possess the density property, (Russian) Mat. Sb. 187 (1996), no. 7, 113-138; translation in Sb. Math. 187 (1996), no. 7, 1061-1085

[10] - On differentiation of integrals with respect to bases of convex sets, Studia Math. 119 (1996), no. 2, 99-108.

[11] M. H. Taibleson, On the theory of Lipschitz spaces of distributions on Euclidean n-space. I. Principal properties, J. Math. Mech. 13 (1964), 407-479.

[12] H. Triebel, Theory of function spaces. II, Monographs in Mathematics, 84. Birkhäuser Verlag, Basel, 1992.

IMAL-CONiCET, Güemes 3450, 3000 Santa Fe, Argentina.

E-mail address: haimar@ceride.gov.ar

E-mail address: lforzani@math.unl.edu.ar

E-mail address: vnaibo@math.unl.edu.ar 